\title{
Simulation of Optical Spatial Filters by Using Fast Fourier Transform
}

\author{
Serkan ALAGOZ ${ }^{1 D}$, Baris Baykant ALAGOZ ${ }^{*}$ \\ ${ }^{1}$ Inonu University, Department of Physics, Malatya, Turkey \\ 2Inonu University, Department of Computer Engineering, Malatya, Turkey \\ (serkan.alagoz@inonu.edu.tr, baykant.alagoz@inonu.edu.tr)
}

\begin{abstract}
Due to the wave nature of the light, harmonic analysis (Fourier transform) and superposition are used in analysis and design of optical systems and Fourier optics experiments. This study aims to illustrate use of fast Fourier transform computations to simulate optical spatial filter results. To design Fourier optics experiments or devices, it is quite useful to perform computer simulations and obtain theoretical results. In this study, fast Fourier transform based simulations for low-pass and high-pass optical spatial filter setups are carried out in the Matlab environment and results of these simulations are reported. Results indicate that this simulation method can be used for the design and analysis of optical spatial filters in addition to the optics education.
\end{abstract}

Keywords : Fourier optics, optical spatial filter, fast Fourier transform,optical system simulation.

\section{Introduction}

Fourier optics and developments in optical systems lead to significant progress in technology and sciences in the last century. Modern physics and astronomy have largely benefited from the results of optics experiments. Although ray optics technique provides useful analysis for basic optical systems, it is not sufficient to explain more advanced topics of optics such as interference, diffraction, resolution, distortion, imaging etc. Therefore, the wave nature of light should be considered in analysis and simulations of optical systems. At this point, harmonic analysis (Fourier transform) and linear system principles (superposition) are employed in optics. Then, Fourier optics can explain Fourier spectrum of objects, transmission of spectral components through optical systems, imaging properties associated with superposition of waves can be analyzed and functional optic devices, which operates on the spectral components, can be realized (Von, 1971; Klein et al, 1987; Stark, 2012; Kedar 2015; Tsegaye, 2019; Macfaden et al, 2017). Then, Fourier transform has become a fundamental tool for optical system simulation (Degallaix, 2010; Day, 2012; O'Neill, 1956). Linear system foundations can be implemented on optical systems and optical spatial filtering has been discussed on the bases of the optical Fourier transform (Stark, 2012; Steward, 1987) and the optical convolution (Kedar 2015; Tsegaye, 2019)

A lens can perform Fourier transform of the monochromatic electromagnetic field that is reflected by an object when an object is at the focal point in front of the lens (O'Neill, 1956; Kedar, 2015). Figure 1 depicts the Fourier plane where the Fourier transform of the field emission from the object plane appears before forming an image of the object on image plane (Tsegaye, 2019; Stark, 2012; Kedar, 2015). One of the promising applications of optical Fourier transform by using a lens is the optical spatial filters. The optical spatial filters enable spatial two-dimensional filtering of an object plane by using optical equipment such as lens and filter masks (O'Neill, 1956; Brown, 1966). However, impurities in optical structure and defects in mask cause additional optic modulations that result in distortion or undesired physical effects (e.g. diffraction or interference patterns) in image plane at the filter output (Tsegaye, 2019). Therefore, before conducting expensive optical experiments in device developments, 
it is important to obtain consistent simulation results in order to better interpret experimental results and limitations of optical systems according to simulation results (Degallaix, 2010; Day, 2012). Applications of optical Fourier transform have been discussed in several works (Castellini et al., 1996, Hoffer at al., 1996, Guan et al. 2016, Palushani at al., 2009)

The current study presents a simulation approach for optical spatial filtering studies. This approach implements two-dimensional fast Fourier transform and inverse fast Fourier transform operations to simulate Fourier optics processes such as optical Fourier transform and image construction on the image plane. The simulation results can show some phenomena of Fourier optics; for instance, diffraction patterns.

\section{Optical Spatial Filtering in Fourier Optics}

Figure 1 depicts an experimental setup that has been used to obtain optical Fourier transform of an object at the object plane (Tsegaye, 2019; Stark, 2012; Kedar 2015). The optical Fourier transform of the object appears at the Fourier plane. These spatial components form an image of the object plane at the image plane, which corresponds to the inverse Fourier transform of spatial distributions that is appearing in the Fourier plane.

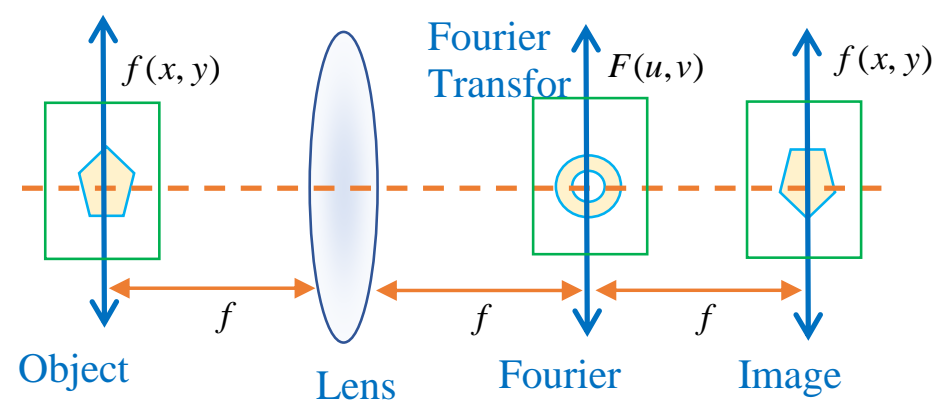

Figure 1. Optical Fourier transform of an object plane at the Fourier plane by using a lens

Let's assume a two dimensional object has a field transmission function $f(x, y)$. The Fourier transform of this object in the Fourier space can be written according to the object's amplitude (Klein et al, 1987; Stark, 2012; Kedar, 2015).

$$
F(u, v)=\frac{1}{(2 \pi)^{2}} \iint f(x, y) e^{i 2 \pi u x+i 2 \pi v y} d x d y,
$$

where parameters $(u, v)$ represents spatial Fourier coordinates. This equation corresponds to continuous Fourier transform of the function $f(x, y)$. For optical spatial filtering with a mask function, a convolution of a mask transfer function $(H(u, v))$ and the object function in Fourier space $(F(u, v))$ is performed in the spatial Fourier domain as (Tsegaye, 2019)

$$
G(u, v)=F(u, v) H(u, v),
$$

where the spatial function $G(u, v)$ represents Fourier transform of the output $g(u, v)$ in the image plane. The $G(u, v)$ is calculated in Fourier space by applying the mask function to spatial components at Fourier plane as in Figure 2. Figure 2 shows a schematic of the spatial optical filtering system (O'Neill, 1956). Optical spatial filtering performs three states: Firstly, spectral components of field transmission function of object $F(u, v)$ is formed at the focal distance of the Lens1 according to optical Fourier transform. Secondly, a filter mask function $H(u, v)$ is applied to these components by using a physical mask sheet. Finally, by using the filter mask, the filtered image of the object is formed at the focal lens of the Lens2, which corresponds to inverse Fourier transform. 


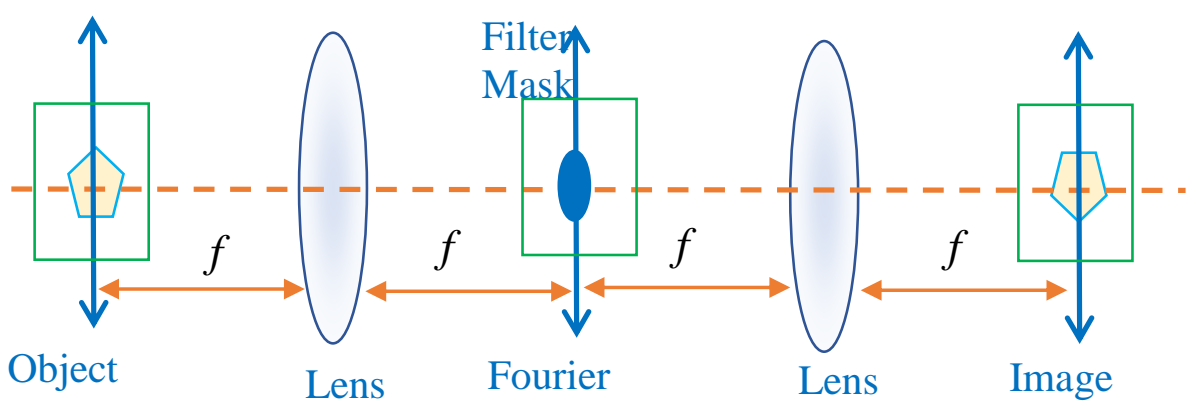

Figure 2. Optical spatial filtering by using two lens and a filter mask at the Fourier plane

\section{Simulation of Optical Spatial Filtering by Fast Fourier Transform}

For the simulation of the optical spatial filtering setup in Figure 2, the following algorithm is employed:

Step 1: Calculate Fourier transform of object plane by using fast Fourier transform. (This step simulates optical Fourier transform at the Lens1.)

Step2: Apply filter mask to spatial components at the Fourier plane by using equation (2). (This step simulates the effects of the filter mask in the Fourier plane.)

Step 3: Calculate inverse Fourier transform of the masked spatial components to obtain filter output in the image plane. (This step simulates image reconstruction on the image plane next to Lens2.)

The following subsections illustrate some simulation results for a low pass spatial filtering and high pass spatial filtering by using fast Fourier transform function in the Matlab.

\subsection{Simulation of Optical Low Pass Spatial Filtering}

Figure 3 shows the object plane with a field reflection of A letter and the low-pass filter mask plane. The object plane image in Figure 3(a) simulates a reflector of A letter, which can be illuminated by a monochromatic light source. The reflected light from this plane goes to Lens1 to form Fourier components of the object A letter on the Fourier plane. At the Fourier plane, a binary mask function, which simulates a circular hole with a radius of $50 \mathrm{~mm}$, is used to filter high frequency components. The low frequency components, which gather at the center, pass through the circular hole and reach the second lens.
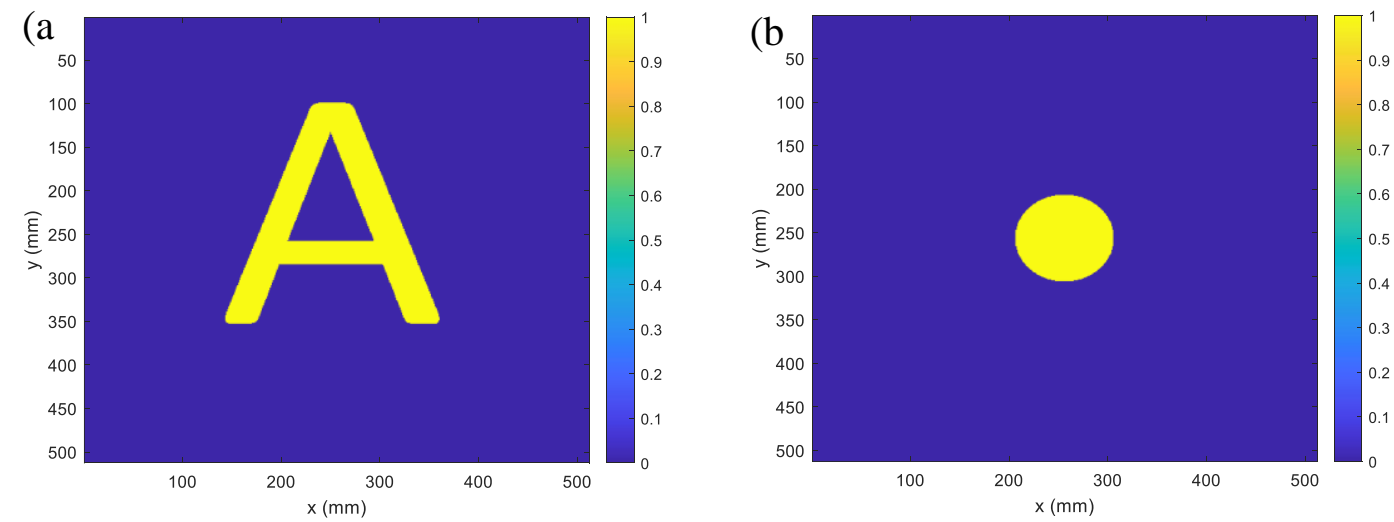

Figure 3. (a) The object of ' $A$ ' letter at the object plane of the spatial filter. (b) The filter mask that allows a passage at the center of the plane for low-frequency components 
Figure 4(a) shows distribution of spatial wave components of the object A letter on the Fourier plane after the low-pass masking occurs. Here, the high frequency components are blockaded with the filter mask in Figure 3(b). Figure 4(b) shows the filter output that is obtained at the image plane. This image appears on the image plane that is at the focal point of Lens2. The figure clearly illustrates low-pass filtering effects on the image.
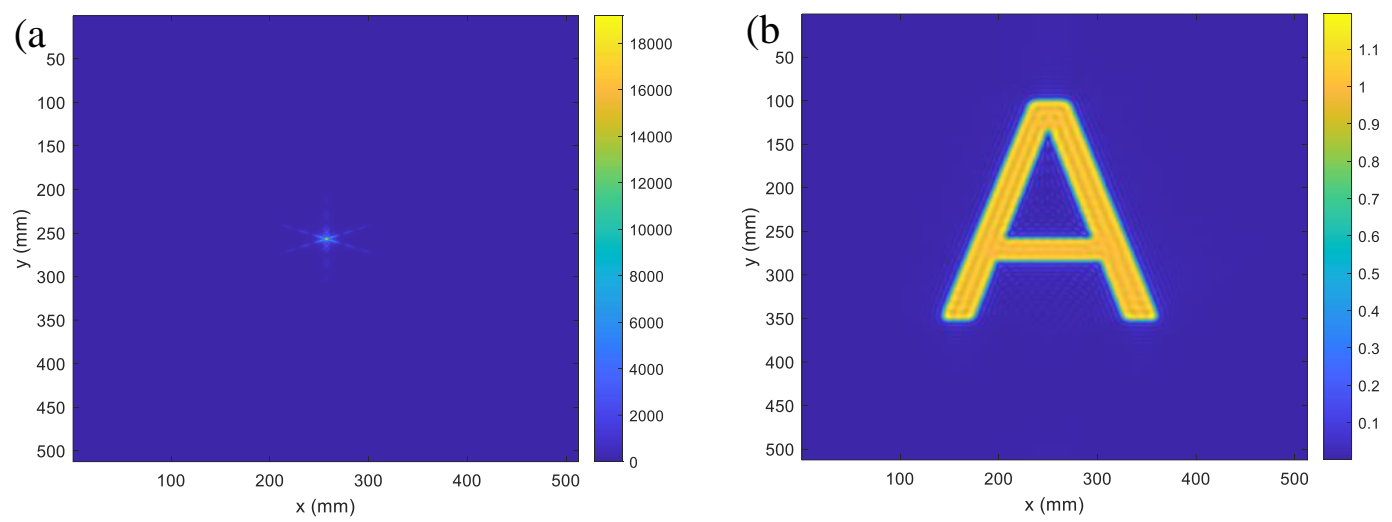

Figure 4. (a) Magnitudes of spatial wave components in Fourier plane for object of 'A' letter after applying the low-pass mask. (b) The filter output that is obtained at the image plane

Figure 5 shows a close of view of the filter output. Diffraction patterns around A letter are observable in simulation results. These patterns are expected physical phenomena that are caused by mask edges that block some spatial components and only allow the low frequency components that are collected at the center of the plane.
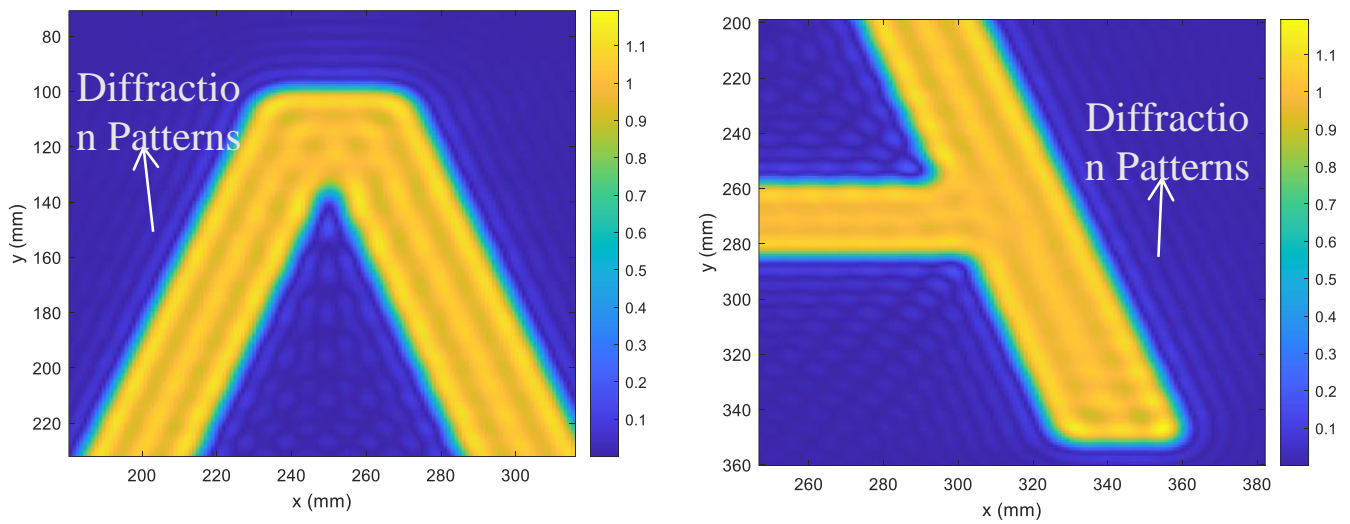

Figure 5. Diffraction patterns around A letter at the image plane

\subsection{Simulation of Optical High Pass Spatial Filtering}

Figure 6 shows the object plane image and the high-pass spatial filter mask. At the Fourier plane, a binary mask function, which is composed of a circular blockade with a radius of $50 \mathrm{~mm}$, is used to filter low frequency spatial components that are collected at the center. The mask value of 0 causes blockading of wave components. The high frequency components can pass through space around the circular blockade and reach the second lens to form the high pass filtered image.

Figure 7(a) shows distribution of spatial wave components on the Fourier plane after a high pass filtering circular mask that is placed at the center of the Fourier plane. This mask blocks the spatial components that coincide to mask blockage (the value of zero). Figure 7(b) shows the filter output that is obtained at the image plane at the focal point of Lens 2 . The figure clearly illustrates high-pass filtering effects on the image $\mathrm{A}$, which amplifies the light intensity at edges because the edges of the image are constructed with the high frequency spatial components. 
Figure 8 shows a close of view of the filter output. Diffraction patterns around the letter A can be observable in simulation results. These patterns are a result of physical phenomenon that is referred to as the diffraction.
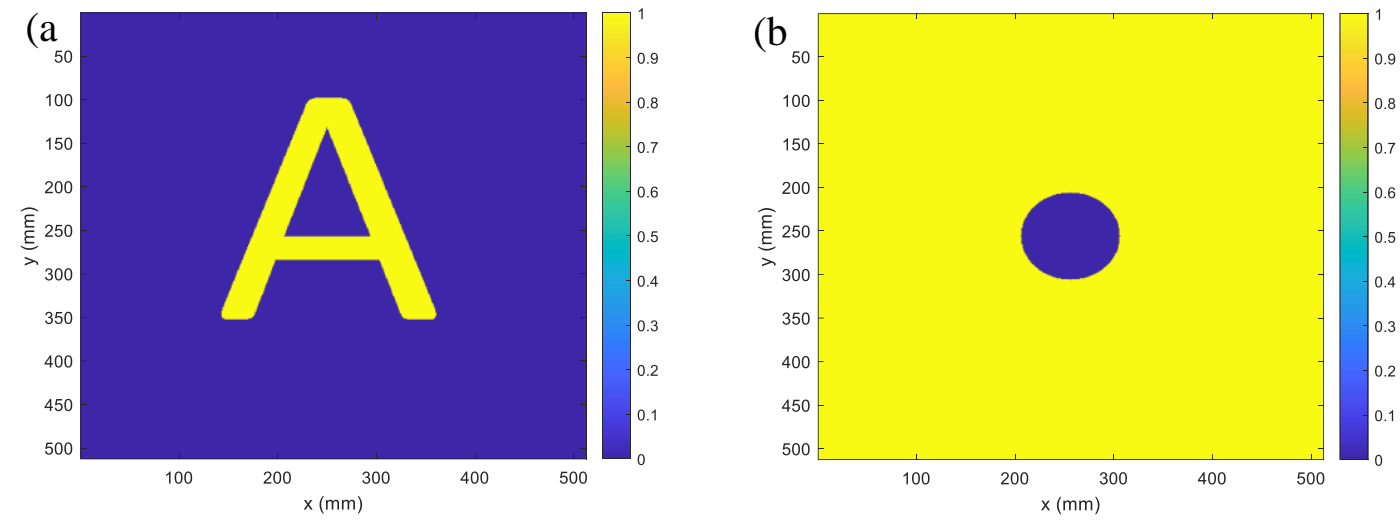

Figure 6. (a) The object of ' $A$ ' letter at the object plane of the spatial filter. (b) The filter mask that allows a passage at the center of plane for low-frequency components
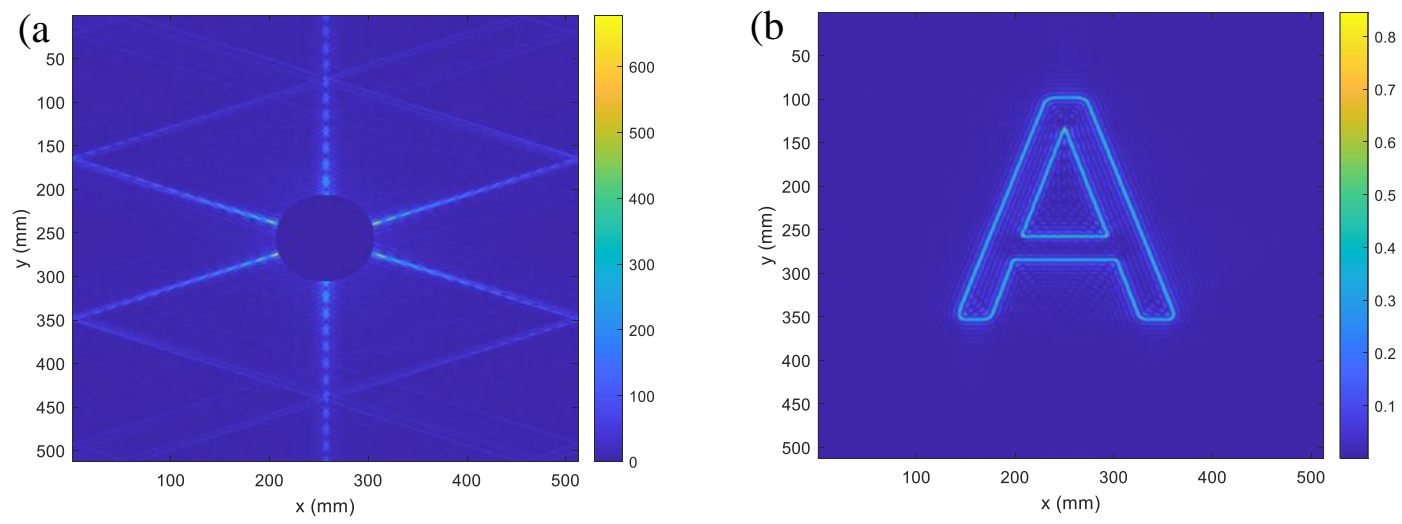

Figure 7. (a) Magnitudes of spatial wave components in the Fourier plane for the object of 'A' letter.

(b) The filter output that is obtained at the image plane
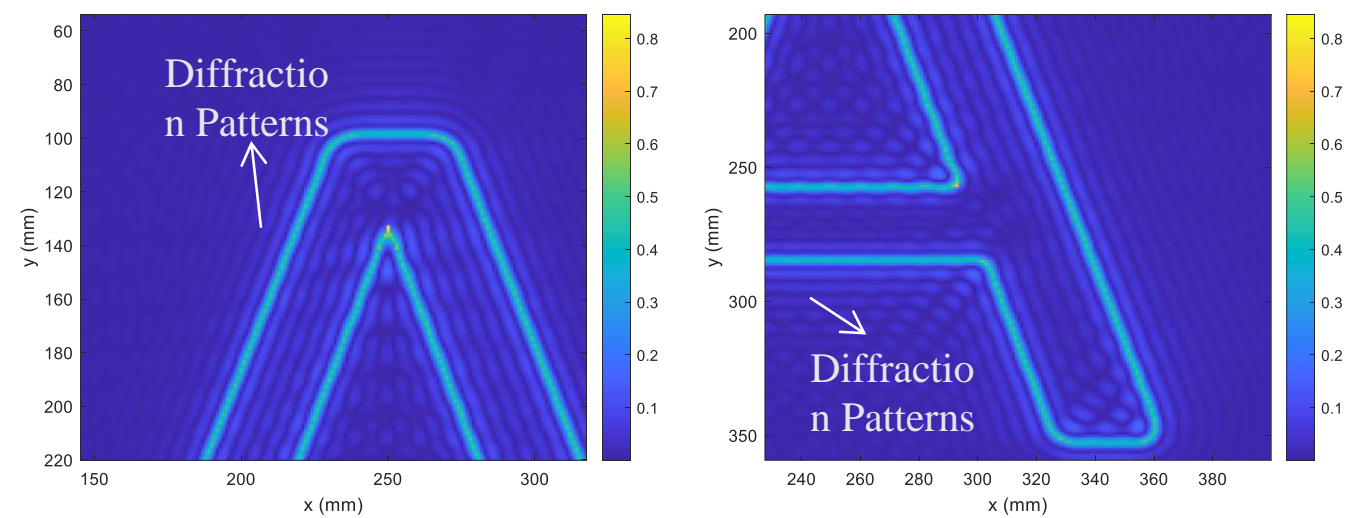

Figure 8. Diffraction patterns around A letter at the image plane. 


\section{Conclusions}

This study illustrated some simulation results for optical spatial filtering experiments. To perform calculations, fft2() and iff2() functions of the Matlab were used. Mask functions for low pass and high pass filtering are formed by binary image with a relevant spatial formation. Results indicated that numerical simulation of the optical spatial filtering experiments can be performed by using this simulation environment. Also, these simulations can be useful for design of optical Fourier filter devices. Previously, Matlab simulations for Fourier optics were implemented in advance design problems (Degallaix, 2010; Day, 2012). This study focused on the simulation of the optical spatial filter experiments (Tsegaye, 2019; Rosen, J. 1997). The results obtained indicate that this simulation scheme can allow considering some physical phenomenon such as diffraction patterns that can be observed in real optical systems. Therefore, the simulation method can be an effective tool for the design of optical spatial filter devices. It can also be used for educational purposes in Fourier optics in order to explain some experimental results.

\section{References}

Brown, B. R., \& Lohmann, A. W. (1966). Complex spatial filtering with binary masks. Applied Optics, 5(6): $967-969$.

Castellini, C., Francini, F., Longobardi, G., Tiribilli, B., \& Sansoni, P. (1996). On-line textile quality control using optical Fourier transforms. Optics and lasers in engineering, 24(1), 19-32.

Day R., A new FFT code: FOG Fast Fourier Transform Optical Simulation of Gravitational Wave Interferometers, Wave Advanced Detector Workshop (GWADW 2012), 2012.

Degallaix, J. (2010). OSCAR a Matlab based optical FFT code. In Journal of Physics: Conference Series, 228(1), p.012021

Guan, P., Røge, K. M., Lillieholm, M., Galili, M., Hu, H., Morioka, T., \& Oxenløwe, L. K. (2016). Time lens-based optical fourier transformation for all-optical signal processing of spectrally-efficient data. Journal of Lightwave Technology, 35(4), 799-806.

Hoffer, L. M., Francini, F., Tiribilli, B., \& Longobardi, G. (1996). Neural networks for the optical recognition of defects in cloth. Optical Engineering, 35(11), 3183-3190.

Kedar K. (2015) Fourier optics and computational imaging. John Wiley \& Sons.

Klein V.M., \& Furtak T.E. (1986) Optics, John Wiley and Sons, New York.

Macfaden, A. J., Gordon, G. S., \& Wilkinson, T. D. (2017). An optical Fourier transform coprocessor with direct phase determination. Scientific reports, 7(1):1-8.

O'Neill, E. (1956). Spatial filtering in optics. IRE Transactions on Information Theory, 2(2):56-65.

Palushani, E., Oxenlowe, L. K., Galili, M., Mulvad, H. C. H., Clausen, A. T., \& Jeppesen, P. (2009). Flat-top pulse generation by the optical Fourier transform technique for ultrahigh speed signal processing. IEEE journal of quantum electronics, 45(11), 1317-1324.

Stark H. (2012) Application of Optical Fourier Transforms. Elsevier, 2012.

Steward, E.G. (1987) Fourier Optics: An Introduction, Dover Publications, New York.

Tsegaye A.A. (2019) Spatial Filtering and Optical Convolution, International Journal of Scientific Research 8(1): 6-8.

Rosen, J. (1997). Three-dimensional optical Fourier transform and correlation. Optics Letters, 22(13), 964-966.

Von B. K. (1971). Lens design for optical Fourier transform systems. Applied optics, 10(12), 27392742. 